\title{
Convergence Rate for Compressible Euler Equations with Damping and Vacuum
}

\author{
FEIMIN HUANG \& RONGHUA PAN
}

\author{
Communicated by C. M. DAFERMOS
}

\begin{abstract}
We study the asymptotic behavior of $L^{\infty}$ weak-entropy solutions to the compressible Euler equations with damping and vacuum. Previous works on this topic are mainly concerned with the case away from the vacuum and small initial data. In the present paper, we prove that the entropy-weak solution strongly converges to the similarity solution of the porous media equations in $L^{p}(R)(2 \leqq p<\infty)$ with decay rates. The initial data can contain vacuum and can be arbitrary large. A new approach is introduced to control the singularity near vacuum for the desired estimates.
\end{abstract}

\section{Introduction}

Compressible Euler equations with damping occur in the mathematical modeling of the motion for the compressible gas flow through a porous medium. The medium induces a friction force, proportional to the linear momentum in the opposite direction. Therefore, in a one-dimensional porous medium, the damped compressible Euler equations express the conservation of mass and the momentum balance as follows:

$$
\begin{aligned}
\rho_{t}+(\rho u)_{x} & =0, \\
(\rho u)_{t}+\left(\rho u^{2}+P(\rho)\right)_{x} & =-\alpha \rho u,
\end{aligned}
$$

with the initial data

$$
\rho(x, 0)=\rho_{0}(x) \geqq 0, m(x, 0)=m_{0}(x),
$$

such that

$$
\left(\rho_{0}(x), m_{0}(x)\right) \rightarrow\left(\rho_{ \pm}, m_{ \pm}\right), \text {as } x \rightarrow \pm \infty \text {. }
$$


Here $\rho, u$ and $P$ denote respectively the density, velocity, and pressure; $m=\rho u$ is the momentum and the constant $\alpha>0$ models friction. In this paper, we consider the polytropic perfect gas where $P(\rho)=P_{0} \rho^{\gamma}, 1<\gamma<3$, with $P_{0}$ a positive constant, and $\gamma$ the adiabatic gas exponent. Without loss of generality, $\alpha$ and $P_{0}$ are normalized to be 1 throughout this paper. It is known that (1.1) is of the hyperbolic type with two characteristic speeds $\lambda_{1}=u-\sqrt{P^{\prime}(\rho)}$ and $\lambda_{2}=u+\sqrt{P^{\prime}(\rho)}$. Furthermore, (1.1) is strictly hyperbolic at the point away from vacuum where two characteristics coincide.

However, in the applications, Darcy's law is used to approximate the momentum equation in system (1.1), and thus we obtain

$$
\begin{aligned}
\rho_{t} & =P(\rho)_{x x}, \\
m & =-P(\rho)_{x},
\end{aligned}
$$

where the second equation is the famous Darcy's law and the first equation is the well-known porous-medium equation. So, it is natural to expect some relationship between system (1.1) and system (1.3). In fact, we have the following conjecture; see [17].

Conjecture. Time asymptotically, the system (1.1) is equivalent to the system (1.3).

The main focus of this study is to prove this conjecture. In this paper, we prove that the $L^{\infty}$ weak-entropy solution with vacuum, selected by the physical entropyflux pair, converge, strongly in $L^{p}(R)$ with decay rates, to the similarity solution of the porous-medium equation, determined uniquely by the end-states and the mass distribution of the initial data. The restriction on the initial data is that both end-states are away from vacuum; see Theorem 1 below for details.

In the case away from vacuum, system (1.1) can be transferred to the $p$-system with damping by changing to the Lagrangian coordinates. The first result concerning the above conjecture is due to HSIAO \& LIU [9, 10] for small smooth solutions away from vacuum based on the derivative estimates and the observation of [23]. Since then, this problem has attracted considerable attentions, see $[8,11,12,22$, 24-26, 29]. However, all of these results are away from vacuum and/or require small smooth initial data. For more references on the $p$-system with damping, we refer to $[3,13,14,19,31]$.

When a vacuum occurs in the solution, the difficulty of the problem is greatly increased. The main difficulties come from the fact that such a problem involves three mechanisms: nonlinear convection, lower-order dissipation of damping and the resonance due to vacuum. The interaction of these three mechanisms makes this problem of both mathematical and physical significance. In addition to the shock formation, there is new singularity in our situation due to vacuum. In fact, LIU \& YANG $[20,21]$ observed that the local smooth solutions of (1.1) blow up in finite time before shock formation. This implies the moving of the interface between the vacuum and the gas. Due to this new singularity, it is very difficult to obtain the solutions with any degree of regularity. This makes (1.1) difficult to understand analytically and makes the construction of effective numerical methods for computing solutions a highly non-trivial problem. Indeed, the only global weak solutions are 
constructed in $L^{\infty}$ space by using the method of compensated compactness; see DiNG, CHEN \& LuO [5] for $1<\gamma \leqq \frac{5}{3}$ and HuANG \& PAN [15] for $1 \leqq \gamma<3$. Thus, to study the large-time behavior of solution of (1.1) with vacuum, it is suitable to consider the $L^{\infty}$ weak solution.

Definition 1. We call $(\rho, m)(x, t) \in L^{\infty}$ an entropy-weak solution of (1.1)-(1.2), if, for any non-negative test function $\phi \in \mathcal{D}\left(\mathbf{R}_{+}^{2}\right)$,

$$
\begin{array}{r}
\iint_{t>0}\left(\rho \phi_{t}+m \phi_{x}\right) d x d t+\int_{\mathbf{R}} \rho_{0}(x) \phi(x, 0) d x=0, \\
\iint_{t>0}\left[m \phi_{t}+\left(\frac{m^{2}}{\rho}+P(\rho)\right) \phi_{x}-m \phi\right] d x d t+\int_{\mathbf{R}} m_{0}(x) \phi(x, 0) d x=0, \\
\iint_{t>0}\left(\eta_{e} \phi_{t}+q_{e} \phi_{x}-\rho u^{2} \phi\right) d x d t+\int_{\mathbf{R}} \eta_{e}(x, 0) \phi(x, 0) d x \geqq 0 .
\end{array}
$$

Here, the entropy-flux pair $\left(\eta_{e}, q_{e}\right)$ is associated with mechanical energy:

$$
\begin{aligned}
& \eta_{e}=\frac{1}{2} \rho u^{2}+\frac{1}{(\gamma-1)} \rho^{\gamma}, \\
& q_{e}=\frac{1}{2} \rho u^{3}+\frac{\gamma}{\gamma-1} \rho^{\gamma} u .
\end{aligned}
$$

Without regularity of the solutions, the framework given by HSIAO \& LIU [9, 10] is no longer applicable. Recently, HUANG \& PAN [15] first proved the conjecture above with vacuum in some sense by using the theory of compensated compactness, together with the entropy estimates and rescaling techniques. This method was developed first by SERRE \& XIAO [28]. However, the result in [15] is far from satisfactory. In fact, the results in [15] only give the $L_{\mathrm{loc}}^{q}(1 \leqq q<\infty)$ convergence of density in the solutions of (1.1) and (1.2) to the self-similar solution of the porous medium equation along the level curve of the diffusive similarity profiles. The large-time behavior of the momentum is not known. Furthermore, there are no decay rates in [15]. We will bridge these gaps in this current paper under the restriction that $\rho_{ \pm}$are positive. For this purpose, the derivative estimates are required. As our solutions live in $L^{\infty}$ space, it is hopeless to get derivative estimates since the derivatives of solutions are undefined. The hopeful estimate is an $L^{p}$ estimate for the solutions themselves. However, the conservation of mass in the system (1.1) indicates the existence of anti-derivatives for some quantities. If we consider the equations for these anti-derivatives, the first-order estimates in the original systems provide the derivative estimates for the new equations. With the help of this idea, ZHU [31] obtained the convergence for a damped $p$-system modeling the elastic materials, based on the energy method for wave equations in [9] due to the parabolic structure of the equations. However, this approach fails to solve our problem because of the degeneracy at vacuum. In the spirit of [31], we explore the hyperbolicity of system (1.1) and employ the entropy analysis for (1.1) itself rather than the energy estimate for the wave equation. Detailed analysis on convection terms near vacuum helps us to establish the uniform estimates for the solutions. This estimate gives the strong convergence results with decay rates. 
We state our main result now. Let $(\rho(x, t), m(x, t))$ be the $L^{\infty}$ weak-entropy solution obtained by vanishing viscosity, and let $\bar{\rho}(\eta)\left(\eta=\frac{x}{\sqrt{t+1}}\right)$ be the similarity solution of

$$
\begin{aligned}
\bar{\rho}_{t} & =P(\bar{\rho})_{x x}, \\
\bar{\rho}( \pm \infty) & =\rho_{ \pm}, \quad \rho_{ \pm}>0 .
\end{aligned}
$$

We define

$$
\begin{aligned}
\bar{m} & =-P(\bar{\rho})_{x}, \\
\hat{\rho}(x, t) & =\left(m_{+}-m_{-}\right) e^{-t} \theta(x), \\
\hat{m}(x, t) & =m_{-} e^{-t}+\left(m_{+}-m_{-}\right) e^{-t} \int_{-\infty}^{x} \theta(\xi) d \xi,
\end{aligned}
$$

where $\theta(x)$ is a smooth function with compact support such that

$$
\int_{-\infty}^{\infty} \theta(x) d x=1
$$

and

$$
\begin{aligned}
y(x, t) & =-\int_{-\infty}^{x}\left(\rho(\xi, t)-\bar{\rho}\left(\xi+x_{0}, t\right)-\hat{\rho}(\xi, t)\right) d \xi \\
z(x, t) & =m(x, t)-\bar{m}\left(x+x_{0}, t\right)-\hat{m}(x, t), \\
y_{0} & =-\int_{-\infty}^{x}\left(\rho_{0}(\xi)-\bar{\rho}\left(\xi+x_{0}\right)-\hat{\rho}(\xi, 0)\right) d \xi \\
z(x, t) & =m_{0}(x)-\bar{m}\left(x+x_{0}\right)-\hat{m}(x, 0) .
\end{aligned}
$$

Here $x_{0}$ is the constant uniquely determined by the following equation:

$$
\int_{-\infty}^{\infty}\left(\rho_{0}(x)-\bar{\rho}\left(x+x_{0}\right)\right) d x=m_{+}-m_{-} .
$$

Then we have the following results.

Theorem 1. Suppose that $y_{0}(x) \in H^{1}, \rho_{0}(x)-\bar{\rho}\left(x+x_{0}\right)-\hat{\rho}(x, 0), m_{0}(x)-$ $\hat{m}(x, 0) \in L^{2} \cap L^{\infty}$. Then there exist constants $C>0$ and $\beta<\frac{1}{4}$ independent of time such that

$$
\begin{gathered}
\int_{-\infty}^{\infty}\left(y^{2}+y_{x}^{2}+y_{t}^{2}\right) d x+\int_{0}^{\infty} \int_{-\infty}^{\infty}\left(y_{x}^{2}+y_{t}^{2}\right) d x d t \leqq C \\
\int_{-\infty}^{\infty}\left(\left|y_{x}\right|^{p}+\left|y_{t}\right|^{p}\right) d x \leqq C(1+t)^{-\beta}, 2 \leqq p<\infty .
\end{gathered}
$$

Therefore,

$$
\begin{aligned}
& \left\|\rho(x, t)-\bar{\rho}\left(x+x_{0}, t\right)\right\|_{L^{p}}+\left\|m(x, t)-\bar{m}\left(x+x_{0}, t\right)\right\|_{L^{p}} \\
& \quad \leqq C(1+t)^{-\beta / p}, \quad 2 \leqq p<\infty .
\end{aligned}
$$


If in addition, $\rho_{-}=\rho_{+}$, then

$$
\int_{-\infty}^{\infty}\left(\left|y_{x}\right|^{p}+\left|y_{t}\right|^{p}\right) d x \leqq C(1+t)^{-1}, 2 \leqq p<\infty .
$$

and

$$
\begin{aligned}
& \left\|\rho(x, t)-\bar{\rho}\left(x+x_{0}, t\right)\right\|_{L^{p}}+\left\|m(x, t)-\bar{m}\left(x+x_{0}, t\right)\right\|_{L^{p}} \\
& \quad \leqq C(1+t)^{-1 / p}, \quad 2 \leqq p<\infty .
\end{aligned}
$$

Remark 1. (1) Theorem 1 implies that the weak-entropy solution $\rho(x, t)$ converges strongly in $L^{P}(R)$ towards the nonlinear diffusive profile $\bar{\rho}$ as $t \rightarrow \infty$. Furthermore, Theorem 1 also infers the strong convergence $m(x, t)$ to $\bar{m}$, which is not clear in [15].

(2) Theorem 2 is valid for any $L^{\infty}$ weak entropy solutions of (1.1) obtained by compensated compactness using a viscosity approximation. The estimates in Theorem 1 are independent of the choice of subsequences of approximate solutions. This is clear in our arguments.

Let us explain the basic ideas of this paper. First, we approximate the equations (1.1) and (1.4) by adding an artificial viscosity to get the smooth approximate solutions $\left(\rho^{\varepsilon}, m^{\varepsilon}\right),\left(\bar{\rho}^{\varepsilon}, \bar{m}^{\varepsilon}\right)$. Then, by careful entropy analysis and an energy method, we get some uniformly estimates for $\left(\rho^{\varepsilon}-\bar{\rho}^{\varepsilon}, m^{\varepsilon}-\bar{m}^{\varepsilon}\right)$. Finally, letting $\varepsilon \rightarrow 0$, we get the desired results by the theory of compensated compactness.

The arrangement of the present paper is as follows. In Section 2, some knowledge on the porous-media equation and its approximation are prepared. In Section 3 , the large-time asymptotic behavior is presented by an energy method, entropy analysis and the theory of compensated compactness.

\section{Diffusive profiles and its approximation}

Consider the following Cauchy problem for the porous-media equation (PME):

$$
\begin{aligned}
\rho_{t} & =\left(\rho^{\gamma}\right)_{x x}, \gamma \geqq 1, t>0, \\
\rho(x, 0) & =\rho_{0}(x)=\rho_{-} \chi(x<0)+\rho_{+} \chi(x>0),
\end{aligned}
$$

where, $\chi$ is the characteristic function and $\rho_{ \pm}$are positive constants. Without loss of generality, we assume that $0<\rho_{+}<\rho_{-}$.

It is clear that the PME is strictly parabolic if $\rho>0$. The problem (2.1) has been extensively studied. We refer to [1, 2, 7, 16, 27]. In [15], HUANG \& PAN have proved the uniqueness of weak solutions with $L^{\infty}$ initial data for PME. Since the initial data is self-similar, the uniqueness theorem of [15] implies that $\rho$ itself is self-similar, i.e., $\rho(x, t)=\bar{\rho}(z)\left(z=\frac{x}{\sqrt{t}}\right)$, which satisfies

$$
\begin{aligned}
\left(\gamma \bar{\rho}^{\gamma-1} \bar{\rho}_{z}\right)_{z}+\frac{1}{2} z \bar{\rho}_{z} & =0, \\
\bar{\rho}(-\infty)=\rho_{-}, \bar{\rho}(+\infty) & =\rho_{+} .
\end{aligned}
$$

The following propositions are due to [1] and [7]. 
Proposition 2.1. There is one and only one solution $\bar{\rho}(z) \in C^{2}$ to (2.2) satisfying the following:

(1) $\rho_{+} \leqq \bar{\rho}(z) \leqq \rho_{-}$is monotone decreasing on $\mathbf{R}$.

(2) $\left(\left|\bar{\rho}_{x}\right|,\left|\bar{\rho}_{t}\right|\right) \leqq C\left(t_{1}\right)\left(t^{-\frac{1}{2}}, t^{-1}\right)$ for any $t \geqq t_{1}>0$.

Now let us approximate the equation (2.1) with viscosity

$$
\begin{aligned}
\bar{\rho}_{t}^{\varepsilon} & =\left(\bar{\rho}^{\varepsilon}\right)_{x x}^{\gamma}+\varepsilon \bar{\rho}_{x x}^{\varepsilon}, \\
\bar{m}^{\varepsilon} & =-P\left(\bar{\rho}^{\varepsilon}\right)_{x}, \\
\bar{\rho}^{\varepsilon}(x, 0) & =\rho_{-} \chi(x<0)+\rho_{+} \chi(x>0) .
\end{aligned}
$$

It is obvious that (2.3) has a unique solution which is self-similar and strictly monotone. It is easy to see that the following properties hold.

Proposition 2.2. The self-similar solution $\bar{\rho}^{\varepsilon}$ of (2.3) satisfies the following dissipative estimates:

$$
\begin{aligned}
\int_{-\infty}^{\infty}\left|\bar{\rho}_{x}^{\varepsilon}\right|^{2} d x & =O(1) t^{-\frac{1}{2}}, \\
\int_{-\infty}^{\infty}\left|\bar{\rho}_{t}^{\varepsilon}\right|^{2}+\left|\bar{\rho}_{x x}^{\varepsilon}\right|^{2} d x & =O(1) t^{-\frac{3}{2}}, \\
\int_{-\infty}^{\infty}\left|\bar{\rho}_{x t}^{\varepsilon}\right|^{2} d x & =O(1) t^{-\frac{5}{2}},
\end{aligned}
$$

where $O(1)$ is a positive constant independent of $\varepsilon$. Furthermore, $\bar{\rho}^{\varepsilon}$ strongly converges to $\bar{\rho}$ as $\varepsilon$ tends to zero, uniformly in $\Omega \subset \subset R \times(0, T)$.

\section{Energy estimates and decay rates}

This section is devoted to the proof of Theorem 1. First of all, we have the following observations.

Lemma 3.1. Let $0 \leqq \rho \leqq M<\infty, 0<a<\bar{\rho} \leqq M<\infty$, there are positive constants $C_{1}$ and $C_{2}$ such that

(1) $(\rho-\bar{\rho})(P(\rho)-P(\bar{\rho})) \geqq C_{1}(\rho-\bar{\rho})^{2}$,

(2) $P(\rho)-P(\bar{\rho})-P^{\prime}(\bar{\rho})(\rho-\bar{\rho}) \geqq C_{2}(\rho-\bar{\rho})^{2}$.

Proof. It is easy to check that Lemma 3.1 holds when $\rho>b>0$, while $C_{1}$ and $C_{2}$ depend on $b$. Thus, to prove Lemma 3.1, it is sufficient to prove it near vacuum.

Choose the constant $\sigma=\frac{a P(a)}{2 M^{2}}$. Let $F(\rho)=(\rho-\bar{\rho})(P(\rho)-P(\bar{\rho}))-\sigma(\rho-\bar{\rho})^{2}$. We have

$$
F(0)=\bar{\rho} P(\bar{\rho})-\sigma \bar{\rho}^{2} \geqq \frac{a P(a)}{2}>0 .
$$

On the other hand, $\left|F^{\prime}(\rho)\right| \leqq C$. Therefore, there exists $s>0$ such that the first inequality holds for any $0 \leqq \rho \leqq s$. This proves the first inequality. 
For the second inequality, we define $H(\rho)=P(\rho)-P(\bar{\rho})-P^{\prime}(\bar{\rho})(\rho-\bar{\rho})-$ $\mu(\rho-\bar{\rho})^{2}$ and choose the constant $\mu=\frac{(\gamma-1) P(a)}{2 M^{2}}$. Then, we have

$$
H(0)=-\bar{\rho}^{\gamma}+\gamma \bar{\rho}^{\gamma}-\mu \bar{\rho}^{2} \geqq \frac{(\gamma-1) P(a)}{2}>0
$$

since $\left|H^{\prime}(\rho)\right| \leqq C$. Thus, there is $r>0$ such that the second inequality holds for any $0 \leqq \rho \leqq r$. We complete the proof of the second inequality.

Let us approximate system (1.1) by adding artificial viscosity with parameter $\varepsilon>0$, i.e.,

$$
\begin{aligned}
\rho_{t}^{\varepsilon}+m_{x}^{\varepsilon} & =\varepsilon \rho_{x x}^{\varepsilon}, \\
m_{t}^{\varepsilon}+\left(\frac{m^{\varepsilon}}{\rho^{\varepsilon}}+P\left(\rho^{\varepsilon}\right)\right)_{x} & =-m^{\varepsilon}+\varepsilon m_{x x}^{\varepsilon} .
\end{aligned}
$$

with initial data

$$
\left(\rho^{\varepsilon}, m^{\varepsilon}\right)(x, 0)=\left(\rho_{0}^{\varepsilon}(x), m_{0}^{\varepsilon}(x)\right)
$$

such that

$$
\begin{array}{r}
\rho_{0}^{\varepsilon}(x)>\varepsilon, \quad \rho_{0}^{\varepsilon}(x) \rightarrow \rho_{ \pm}, \text {as } x \rightarrow \pm \infty, \\
\rho_{0}^{\varepsilon}(x) \rightarrow \rho_{0}(x), \text { and } m_{0}^{\varepsilon}(x) \rightarrow m_{0}(x), \text { a.e., as } \varepsilon \rightarrow 0 .
\end{array}
$$

It is easy to check that there is a global smooth solution $\left(\rho^{\varepsilon}, m^{\varepsilon}\right)$ with $\rho^{\varepsilon}>0$ for (3.1), (3.2) due to DIPERNA [6]. We note that for the homogeneous case of (1.1), the existence of weak-entropy solution is proved by DING, CHENG \& LUO [4] for $1<\gamma \leqq \frac{5}{3}$ and LIONS, PERTHAME \& SOUGANIDIS [18] for $\frac{5}{3}<\gamma<3$. For the nonhomogeneous case of (1.1), DING, CHENG \& LUO [5] also have proved the global existence of (1.1), (1.2) for $1<\gamma \leqq \frac{5}{3}$ by the Lax-Friedrichs scheme. Therefore, by the convergence theorems of $[4,5,18]$, we can assume that as $\varepsilon \rightarrow 0,\left(\rho^{\varepsilon}, m^{\varepsilon}\right)$ converges almost everywhere to $(\rho, m)$ which is a weak-entropy solution of (1.1), (1.2); see [15].

Let $\left(\bar{\rho}^{\varepsilon}, \bar{m}^{\varepsilon}\right)$ be the smooth solution of $(2.3)$ and

$$
\begin{aligned}
& w=\rho^{\varepsilon}-\bar{\rho}^{\varepsilon}, \\
& z=m^{\varepsilon}-\bar{m}^{\varepsilon} .
\end{aligned}
$$

Then we have

$$
\begin{aligned}
w_{t}+z_{x} & =\varepsilon w_{x x} \\
z_{t}+\left(\frac{m^{\varepsilon}}{\rho^{\varepsilon}}\right)_{x}+\left(P\left(\rho^{\varepsilon}\right)-P\left(\bar{\rho}^{\varepsilon}\right)\right)_{x}+z & =\varepsilon z_{x x}-\bar{m}_{t}^{\varepsilon}+\varepsilon \bar{m}_{x x}^{\varepsilon} .
\end{aligned}
$$

Integrating the first equation of (3.3), we have

$$
\frac{d}{d t} \int_{-\infty}^{\infty}\left(\rho^{\varepsilon}-\bar{\rho}^{\varepsilon}\right) d x=e^{-t}\left(m_{+}-m_{-}\right),
$$


due to the fact that $m^{\varepsilon}( \pm \infty, t)=e^{-t} m_{ \pm}$. This yields

$$
\frac{d}{d t} \int_{-\infty}^{\infty}\left(\rho^{\varepsilon}(x, t)-\bar{\rho}^{\varepsilon}\left(x+x_{0}^{\varepsilon}, t\right)-\hat{\rho}(x, t)\right) d x=0,
$$

where $x_{0}^{\varepsilon}$ is determined by

$$
\int_{-\infty}^{\infty}\left(\rho_{0}^{\varepsilon}(x)-\bar{\rho}^{\varepsilon}\left(x+x_{0}^{\varepsilon}, 0\right)\right) d x=m_{+}-m_{-},
$$

and satisfies $x_{0}^{\varepsilon} \rightarrow x_{0}$, as $\varepsilon \rightarrow 0$.

We remark that $\hat{\rho}$ and $\hat{m}$ decay exponentially. For convenience, we only prove the case for $m_{+}=m_{-}=0$ in this paper. Other cases can be easily treated in the similar way.

Thus, we have

$$
\int_{-\infty}^{\infty}\left(\rho_{0}^{\varepsilon}(x)-\bar{\rho}^{\varepsilon}\left(x+x_{0}^{\varepsilon}, 0\right)\right) d x=0,
$$

and we can define $y=-\int_{-\infty}^{x} w(r, t) d r$ so that

$$
y_{x}=-w, \quad z=y_{t}-\varepsilon y_{x x} .
$$

Multiplying $y$ with the second equation of (3.3), integrating over $(-\infty, \infty) \times$ $[0, t]$, by Lemma 3.1 , we get

$$
\begin{aligned}
\int_{-\infty}^{\infty} & \left(z y+\frac{1}{2} y^{2}+\varepsilon y_{x}^{2}\right) d x-\int_{0}^{t} \int_{-\infty}^{\infty} z^{2} d x d \tau \\
& +\int_{0}^{t} \int_{-\infty}^{\infty} 2 \varepsilon^{2} y_{x x}^{2}+\left(C_{1}+\varepsilon\right) y_{x}^{2} d x d \tau-\int_{0}^{t} \int_{-\infty}^{\infty} \frac{m^{\varepsilon}}{\rho^{\varepsilon}} y_{x} d x d \tau \\
\leqq & C\left(\left\|y_{0}\right\|^{2}+\left\|z_{0}\right\|^{2}\right)+\varepsilon\left\|y_{0 x}\right\|^{2} \\
& +C\left|\int_{0}^{t} \int_{-\infty}^{\infty} \bar{\rho}_{t}^{\varepsilon} y_{x} d x d \tau\right|+C\left|\int_{0}^{t} \int_{-\infty}^{\infty} \bar{\rho}_{x x}^{\varepsilon} y_{x} d x d \tau\right| .
\end{aligned}
$$

We compute

$$
\begin{aligned}
& \left|\int_{0}^{t} \int_{-\infty}^{\infty} \bar{\rho}_{t}^{\varepsilon} y_{x} d x d \tau\right|+\left|\int_{0}^{t} \int_{-\infty}^{\infty} \bar{\rho}_{x x}^{\varepsilon} y_{x} d x d \tau\right| \\
& \quad \leqq \int_{0}^{t} \int_{-\infty}^{\infty}\left(\left(\bar{\rho}_{t}^{\varepsilon}\right)^{2}+\left(\bar{\rho}_{x x}^{\varepsilon}\right)^{2}\right) d x d \tau+\delta \int_{0}^{t} \int_{-\infty}^{\infty} y_{x}^{2} d x d \tau \\
& \quad \leqq C+\delta \int_{0}^{t} \int_{-\infty}^{\infty} y_{x}^{2} d x d \tau .
\end{aligned}
$$

On the other hand, since

$$
\begin{aligned}
\frac{\left(m^{\varepsilon}\right)^{2}}{\rho^{\varepsilon}} & =\frac{\left(\bar{m}^{\varepsilon}\right)^{2}}{\bar{\rho}^{\varepsilon}}+\frac{\left(m^{\varepsilon}\right)^{2}-\left(\bar{m}^{\varepsilon}\right)^{2}}{\bar{\rho}^{\varepsilon}}+\left(m^{\varepsilon}\right)^{2}\left(\frac{1}{\rho^{\varepsilon}}-\frac{1}{\bar{\rho}^{\varepsilon}}\right) \\
& =\frac{\left(\bar{m}^{\varepsilon}\right)^{2}}{\bar{\rho}^{\varepsilon}}+\frac{\left(m^{\varepsilon}\right)^{2}}{\rho^{\varepsilon} \bar{\rho}^{\varepsilon}} y_{x}+\frac{\left(z^{2}+2 \bar{m}^{\varepsilon} z\right)}{\bar{\rho}^{\varepsilon}}
\end{aligned}
$$


we have

$$
\begin{aligned}
&\left|\int_{0}^{t} \int_{-\infty}^{\infty} \frac{m^{\varepsilon}}{\rho^{\varepsilon}} y_{x} d x d \tau\right| \\
& \leqq\left|\int_{0}^{t} \int_{-\infty}^{\infty} \frac{\left(\bar{m}^{\varepsilon}\right)^{2}}{\bar{\rho}^{\varepsilon}} y_{x} d x d \tau\right|+\int_{0}^{t} \int_{-\infty}^{\infty} \frac{m^{\varepsilon}}{\rho^{\varepsilon} \bar{\rho}^{\varepsilon}} y_{x}^{2} d x d \tau \\
&+\left|\int_{0}^{t} \int_{-\infty}^{\infty} \frac{\left(z^{2}+2 \bar{m}^{\varepsilon} z\right)}{\bar{\rho}^{\varepsilon}} y_{x} d x d \tau\right| \\
& \leqq C(\delta)+\delta \int_{0}^{t} \int_{-\infty}^{\infty} y_{x}^{2} d x d \tau+C(\delta) \int_{0}^{t} \int_{-\infty}^{\infty} z^{2} d x d \tau \\
&+C \int_{0}^{t} \int_{-\infty}^{\infty}\left|\bar{\rho}_{x}^{\varepsilon}\right|^{2} y_{x}^{2} d x d t \\
& \leqq C(\delta)+C \delta \int_{0}^{t} \int_{-\infty}^{\infty} y_{x}^{2} d x d \tau+C(\delta) \int_{0}^{t} \int_{-\infty}^{\infty} z^{2} d x d \tau
\end{aligned}
$$

where we have used Propositions 2.1 and 2.2, Lemma 3.1, and the fact that

$$
\begin{aligned}
& \int_{0}^{t} \int_{-\infty}^{\infty} \frac{m^{\varepsilon}}{\rho^{\varepsilon} \bar{\rho}^{\varepsilon}} y_{x}^{2} d x d \tau \\
& \quad \leqq C \int_{0}^{t} \int_{-\infty}^{\infty}\left(|z| y_{x}^{2}+\left|\bar{m}^{\varepsilon}\right| y_{x}^{2}\right) d x d \tau \\
& \quad \leqq \int_{0}^{t} \int_{-\infty}^{\infty}\left(\delta y_{x}^{2}+C(\delta) z^{2}\right) d x d \tau+\int_{0}^{t} \int_{-\infty}^{\infty}\left(\delta\left|y_{x}\right|^{\frac{8}{3}}+C(\delta)\left(\bar{\rho}_{x}^{\varepsilon}\right)^{4}\right) d x d \tau \\
& \quad \leqq C(\delta)+C \delta \int_{0}^{t} \int_{-\infty}^{\infty} y_{x}^{2} d x d \tau+C(\delta) \int_{0}^{t} \int_{-\infty}^{\infty} z^{2} d x d \tau
\end{aligned}
$$

Thus, by choosing $\delta$ suitably small, we conclude that, from (3.4) and (3.7), there are positive constants $C_{4}, C_{5}$ and $C_{6}$ such that

$$
\begin{aligned}
& \int_{-\infty}^{\infty}\left(z y+\frac{1}{2} y^{2}+\varepsilon y_{x}^{2}\right) d x+C_{4} \int_{0}^{t} \int_{-\infty}^{\infty} y_{x} d x d \tau-C_{5} \int_{0}^{t} \int_{-\infty}^{\infty} z^{2} d x d \tau \\
& \quad \leqq C_{6} .
\end{aligned}
$$

Let $\eta_{e}^{\varepsilon}$ be the mechanical energy and $q^{\varepsilon}$ the related flux. We denote by $\eta_{*}$ the quantity

$$
\eta_{e}^{\varepsilon}-\frac{1}{\gamma-1} P^{\prime}\left(\bar{\rho}^{\varepsilon}\right)\left(\rho^{\varepsilon}-\bar{\rho}^{\varepsilon}\right)-\frac{1}{\gamma-1} P\left(\bar{\rho}^{\varepsilon}\right) .
$$

It is easy to check that

$$
\frac{1}{\gamma-1} P\left(\bar{\rho}^{\varepsilon}\right)_{x}=-\frac{\left(\bar{m}^{\varepsilon}\right)^{2}}{\bar{\rho}^{\varepsilon}}
$$




$$
\begin{aligned}
& \frac{d}{d t} \int_{-\infty}^{\infty} \eta_{*}(x, t) d x+\frac{d}{d t} \int_{-\infty}^{\infty} \frac{1}{\gamma-1}\left[P^{\prime}\left(\bar{\rho}^{\varepsilon}\right)\left(\rho^{\varepsilon}-\bar{\rho}^{\varepsilon}\right)\right] d x \\
& \quad+\int_{-\infty}^{\infty}\left(\frac{m^{\varepsilon}}{\rho^{\varepsilon}}-\frac{\left(\bar{m}^{\varepsilon}\right)^{2}}{\bar{\rho}^{\varepsilon}}\right) d x \leqq \varepsilon \int_{-\infty}^{\infty} \gamma\left(\bar{\rho}^{\varepsilon}\right)^{\gamma-2}\left(\bar{\rho}_{x}^{\varepsilon}\right)^{2} d x \leqq C \varepsilon
\end{aligned}
$$

due to the convexity of $\eta_{e}^{\varepsilon}$ and entropy inequality. Integrating (3.11) over [0,t], we have

$$
\begin{gathered}
\int_{-\infty}^{\infty} \eta_{*}(x, t) d x+\int_{0}^{t} \int_{-\infty}^{\infty}\left(\frac{m^{\varepsilon} 2}{\rho^{\varepsilon}}-\frac{\left(\bar{m}^{\varepsilon}\right)^{2}}{\bar{\rho}^{\varepsilon}}\right) d x d \tau \\
\leqq C+\delta \int_{-\infty}^{\infty} y^{2}(x, t) d x+C \varepsilon t
\end{gathered}
$$

where we have used

$$
\begin{aligned}
\left|\int_{-\infty}^{\infty} \frac{1}{\gamma-1}\left[P^{\prime}\left(\bar{\rho}^{\varepsilon}\right)\left(\rho^{\varepsilon}-\bar{\rho}^{\varepsilon}\right)\right] d x\right| & \leqq C\left|\int_{-\infty}^{\infty} \bar{\rho}_{x}^{\varepsilon} y d x\right| \\
& \leqq C(\delta)+\delta \int_{-\infty}^{\infty} y^{2}(x, t) d x
\end{aligned}
$$

We now make the Taylor's expansion of $\frac{\left(m^{\varepsilon}\right)^{2}}{\rho^{\varepsilon}}$ around $\frac{\left(\bar{m}^{\varepsilon}\right)^{2}}{\bar{\rho}^{\varepsilon}}$ as follows:

$$
\frac{m^{\varepsilon}}{\rho^{\varepsilon}}=\frac{\left(\bar{m}^{\varepsilon}\right)^{2}}{\bar{\rho}^{\varepsilon}}+\frac{2 \bar{m}^{\varepsilon}}{\bar{\rho}^{\varepsilon}} z-\frac{\left(\bar{m}^{\varepsilon}\right)^{2}}{\left(\bar{\rho}^{\varepsilon}\right)^{2}}\left(\rho^{\varepsilon}-\bar{\rho}^{\varepsilon}\right)+Q,
$$

where

$$
Q=\frac{\left(m^{\varepsilon}\right)^{2}}{\rho^{\varepsilon}}-\frac{2 \bar{m}^{\varepsilon}}{\bar{\rho}^{\varepsilon}} m+\frac{\left(\bar{m}^{\varepsilon}\right)^{2}}{\left(\bar{\rho}^{\varepsilon}\right)^{2}} \rho \geqq 0,
$$

due to the convexity of $\frac{m^{\varepsilon}}{\rho^{\varepsilon}}$. Therefore we have

$$
\frac{m^{\varepsilon}}{\rho^{\varepsilon}}-\frac{\left(\bar{m}^{\varepsilon}\right)^{2}}{\bar{\rho}^{\varepsilon}}=Q+\frac{2 \bar{m}^{\varepsilon}}{\bar{\rho}^{\varepsilon}} z+\frac{\left(\bar{m}^{\varepsilon}\right)^{2}}{\left(\bar{\rho}^{\varepsilon}\right)^{2}} y_{x} .
$$

Since

$$
\left|\frac{\left(\bar{m}^{\varepsilon}\right)^{2}}{\left(\bar{\rho}^{\varepsilon}\right)^{2}} y_{x}\right| \leqq \delta y_{x}^{2}+C(\delta)\left(\bar{\rho}_{x}^{\varepsilon}\right)^{4}
$$

and

$$
\begin{aligned}
\frac{\bar{m}^{\varepsilon}}{\bar{\rho}^{\varepsilon}} z & =f\left(\bar{\rho}^{\varepsilon}\right)_{x}\left(y_{t}-\varepsilon y_{x x}\right) \\
& =-\left(f_{t} y+\varepsilon f_{x} y_{x}\right)_{x}+\left(f_{x} y\right)_{t}+f_{t} y_{x}+\varepsilon y_{x} f_{x x},
\end{aligned}
$$

we have

$$
\begin{aligned}
& \int_{-\infty}^{\infty} \eta_{*}(x, t) d x+\int_{0}^{t} \int_{-\infty}^{\infty} Q d x d \tau \\
& \quad \leqq C(\delta)+C \delta \int_{0}^{t} \int_{-\infty}^{\infty} y_{x}^{2} d x d \tau+C \delta \int_{-\infty}^{\infty} y^{2}(x, t) d x+C \varepsilon t
\end{aligned}
$$


On the other hand, (3.14) implies

$$
\begin{aligned}
\frac{\left(m^{\varepsilon}\right)^{2}}{\rho^{\varepsilon}}-\frac{\left(\bar{m}^{\varepsilon}\right)^{2}}{\bar{\rho}^{\varepsilon}} & =\frac{\left(m^{\varepsilon}\right)^{2}}{\rho^{\varepsilon} \bar{\rho}^{\varepsilon}} y_{x}+\frac{1}{\bar{\rho}^{\varepsilon}}\left(\left(m^{\varepsilon}\right)^{2}-\left(\bar{m}^{\varepsilon}\right)^{2}\right) \\
& =\frac{z^{2}}{\bar{\rho}^{\varepsilon}}+\frac{2 z \bar{m}^{\varepsilon}}{\bar{\rho}^{\varepsilon}}+O(1)\left(Q+\bar{\rho}_{x}^{\varepsilon} z y_{x}+\left(\bar{\rho}_{x}^{\varepsilon}\right)^{2} y_{x}\right) .
\end{aligned}
$$

Note that

$$
\bar{\rho}_{x}^{\varepsilon} z y_{x} \leqq \frac{z^{2}}{2 \bar{\rho}^{\varepsilon}}+\delta y_{x}^{4}+C(\delta)\left(\bar{\rho}_{x}^{\varepsilon}\right)^{4} .
$$

Thus, by(3.12), (3.15), (3.16) and Lemma 3.1, we have

$$
\begin{aligned}
& \int_{-\infty}^{\infty}\left(\frac{m^{\varepsilon}}{2 \rho^{\varepsilon}}+y_{x}^{2}\right) d x+\int_{0}^{t} \int_{-\infty}^{\infty} z^{2} d x d \tau \\
& \quad \leqq C(\delta)+C \delta \int_{0}^{t} \int_{-\infty}^{\infty} y_{x}^{2}+C \delta \int_{-\infty}^{\infty} y^{2}(x, t) d x+C \varepsilon t .
\end{aligned}
$$

Now (3.6) implies

$$
\begin{aligned}
\int_{-\infty}^{\infty} \frac{z^{2}}{2 \bar{\rho}^{\varepsilon}} d x \leqq & C \int_{-\infty}^{\infty}\left(\frac{\left(m^{\varepsilon}\right)^{2}}{\rho^{\varepsilon}}+\frac{\left(\bar{m}^{\varepsilon}\right)^{2}}{\bar{\rho}^{\varepsilon}}\right) d x+\int_{-\infty}^{\infty} \frac{\left(m^{\varepsilon}\right)^{2}}{\rho^{\varepsilon} \bar{\rho}^{\varepsilon}}\left|y_{x}\right| d x \\
& +\left|\int_{-\infty}^{\infty} \frac{2 z \bar{m}^{\varepsilon}}{\bar{\rho}^{\varepsilon}} d x\right| \\
\leqq & C+C \int_{-\infty}^{\infty} \frac{\left(m^{\varepsilon}\right)^{2}}{\rho^{\varepsilon}} d x+\frac{1}{2} \int_{-\infty}^{\infty} \frac{z^{2}}{2 \bar{\rho}^{\varepsilon}} d x
\end{aligned}
$$

hence

$$
\int_{-\infty}^{\infty} z^{2} d x \leqq C+C \int_{-\infty}^{\infty} \frac{\left(m^{\varepsilon}\right)^{2}}{\rho^{\varepsilon}} d x .
$$

Thus, from (3.18), we have

$$
\begin{aligned}
& \int_{-\infty}^{\infty}\left(z^{2}+y_{x}^{2}\right) d x+\int_{0}^{t} \int_{-\infty}^{\infty} z^{2} d x d t \\
& \quad \leqq C(\delta)+C \delta \int_{0}^{t} \int_{-\infty}^{\infty} y_{x}^{2}+C \delta \int_{-\infty}^{\infty} y^{2}(x, t) d x+C \varepsilon t
\end{aligned}
$$

We multiply (3.19) by $N=2\left(C_{4}+C_{5}+1\right)$ and add the result to (3.9), by choosing $\delta$ suitably small, we arrive at

Lemma 3.2. Let the assumptions in Theorem 1 hold. Then

$$
\left\|\left(y, y_{x}, z\right)(\cdot, t)\right\|^{2}+\int_{0}^{t}\left\|\left(y_{x}, z\right)(\cdot, \tau)\right\|^{2} d \tau \leqq C+C \varepsilon t .
$$


Lemma 3.3. Under the conditions of Theorem 1, there is a positive constant $C$ independent of time such that, for any $t \geqq 0$,

$$
\|(\rho-\bar{\rho}, m-\bar{m})(\cdot, t)\|^{2}+\int_{0}^{t}\|(\rho-\bar{\rho}, m-\bar{m})(\cdot, \tau)\|^{2} d \tau \leqq C .
$$

Proof. Due to the proof of Lemma 3.2, for any fixed $T>0$, we know that, for $0<t \leqq T$,

$$
\left\|\left(y, y_{x}, z\right)(\cdot, t)\right\|^{2}+\int_{0}^{t}\left\|\left(y_{x}, z\right)(\cdot, \tau)\right\|^{2} d \tau \leqq C+C \varepsilon T .
$$

Since $y_{x}=-\left(\rho^{\varepsilon}-\bar{\rho}^{\varepsilon}\right), z=\left(m^{\varepsilon}-\bar{m}^{\varepsilon}\right)$, due to the convergence of $\rho^{\varepsilon}, \bar{\rho}^{\varepsilon}, m^{\varepsilon}$ and $\bar{m}^{\varepsilon}$, we have, letting $\varepsilon \rightarrow 0$,

$$
\|(\rho-\bar{\rho}, m-\bar{m})(\cdot, t)\|^{2}+\int_{0}^{t}\|(\rho-\bar{\rho}, m-\bar{m})(\cdot, \tau)\|^{2} d \tau \leqq C
$$

for any $0 \leqq t \leqq T$. Then, a standard continuity argument implies Lemma 3.3.

We now prove the decay estimates. In fact, we have the following Lemma.

Lemma 3.4. Under the conditions of Theorem 1, there is a constant $0<\alpha<\frac{1}{4}$, such that

$$
(1+t)^{\alpha}\left\|\left(y_{x}, z\right)(\cdot, t)\right\|^{2} \leqq C+C \varepsilon(1+t)^{1+\alpha} .
$$

Proof. Multiplying (3.11) by $(1+t)^{\alpha}$, with $0<\alpha<\frac{1}{4}$, we have

$$
\begin{gathered}
(1+t)^{\alpha} \frac{d}{d t} \int_{-\infty}^{\infty} \eta_{*}(x, t) d x+(1+t)^{\alpha} \frac{d}{d t} \int_{-\infty}^{\infty} \frac{1}{\gamma-1}\left[P^{\prime}\left(\bar{\rho}^{\varepsilon}\right)\left(\rho^{\varepsilon}-\bar{\rho}^{\varepsilon}\right)\right] d x \\
+(1+t)^{\alpha} \int_{-\infty}^{\infty}\left(\frac{m^{\varepsilon}}{\rho^{\varepsilon}}-\frac{\left(\bar{m}^{\varepsilon}\right)^{2}}{\bar{\rho}^{\varepsilon}}\right) d x \leqq C \varepsilon(1+t)^{\alpha}
\end{gathered}
$$

This gives

$$
\begin{aligned}
& \frac{d}{d t} \int_{-\infty}^{\infty}(1+t)^{\alpha} \eta_{*}(x, t) d x-\alpha(1+t)^{\alpha-1} \int_{-\infty}^{\infty} \eta_{*}(x, t) \\
& \quad+\frac{d}{d t} \int_{-\infty}^{\infty}(1+t)^{\alpha} \frac{1}{\gamma-1}\left[P^{\prime}\left(\bar{\rho}^{\varepsilon}\right)\left(\rho^{\varepsilon}-\bar{\rho}^{\varepsilon}\right)\right] d x \\
& \quad-\alpha \int_{-\infty}^{\infty}(1+t)^{\alpha-1} \frac{1}{\gamma-1}\left[P^{\prime}\left(\bar{\rho}^{\varepsilon}\right)\left(\rho^{\varepsilon}-\bar{\rho}^{\varepsilon}\right)\right] d x \\
& \quad+\int_{-\infty}^{\infty}(1+t)^{\alpha}\left(\frac{m^{\varepsilon}}{\rho^{\varepsilon}}-\frac{\left(\bar{m}^{\varepsilon}\right)^{2}}{\bar{\rho}^{\varepsilon}}\right) d x \leqq C \varepsilon(1+t)^{\alpha} .
\end{aligned}
$$


Integrating (3.22) over $(0, t)$, we have

$$
\begin{aligned}
& \int_{-\infty}^{\infty}(1+t)^{\alpha} \eta_{*}(x, t) d x+\int_{-\infty}^{\infty}(1+t)^{\alpha} \frac{1}{\gamma-1}\left[P^{\prime}\left(\bar{\rho}^{\varepsilon}\right)\left(\rho^{\varepsilon}-\bar{\rho}^{\varepsilon}\right)\right] d x \\
& \quad+\int_{-\infty}^{\infty}(1+\tau)^{\alpha}\left(\frac{\left(m^{\varepsilon}\right)^{2}}{\rho^{\varepsilon}}-\frac{\left(\bar{m}^{\varepsilon}\right)^{2}}{\bar{\rho}^{\varepsilon}}\right) d x d \tau \\
& \leqq C+C \varepsilon(1+t)^{\alpha+1}+\alpha \int_{0}^{t} \int_{-\infty}^{\infty}(1+\tau)^{\alpha-1} \eta_{*}(x, t) d x d \tau \\
& \quad+\frac{\alpha}{\gamma-1} \int_{0}^{t} \int_{-\infty}^{\infty}(1+\tau)^{\alpha-1}\left[P^{\prime}\left(\bar{\rho}^{\varepsilon}\right)\left(\rho^{\varepsilon}-\bar{\rho}^{\varepsilon}\right)\right] d x d \tau
\end{aligned}
$$

Since $\alpha<\frac{1}{4}$, we have

$$
\begin{aligned}
\left|\int_{-\infty}^{\infty}(1+t)^{\alpha} \bar{\rho}_{x}^{\varepsilon} y d x\right| & \leqq \int_{-\infty}^{\infty}(1+t)^{2 \alpha}\left(\bar{\rho}_{x}^{\varepsilon}\right)^{2} d x+\int_{-\infty}^{\infty} y^{2} d x \\
& \leqq C+C \varepsilon t
\end{aligned}
$$

and

$$
\begin{aligned}
& \int_{0}^{t} \int_{-\infty}^{\infty}(1+\tau)^{\alpha-1} \bar{\rho}_{x}^{\varepsilon} y d x d t \\
& \quad \leqq \int_{0}^{t} \int_{-\infty}^{\infty}(1+\tau)^{2 \alpha-2+1+\delta}\left(\bar{\rho}_{x}^{\varepsilon}\right)^{2} d x d t+\int_{0}^{t}(1+\tau)^{-1-\delta}\|y\|_{L^{2}}^{2} d x \\
& \quad \leqq C \int_{0}^{t}(1+t)^{2 \alpha-1+\varepsilon-\frac{1}{2}} d t+C \int_{0}^{t}(1+t)^{-1-\delta}(1+C \varepsilon t) d t \\
& \quad \leqq C+C \varepsilon t
\end{aligned}
$$

By Lemma 3.2, we have, for sufficiently small $\delta$,

$$
\begin{aligned}
& \int_{-\infty}^{\infty}(1+t)^{\alpha} \eta_{*} d x+\int_{0}^{t} \int_{-\infty}^{\infty}(1+\tau)^{\alpha}\left(\frac{m^{\varepsilon}}{\rho^{\varepsilon}}-\frac{\bar{m}^{\varepsilon^{2}}}{\bar{\rho}^{\varepsilon}}\right) d x d \tau \\
& \leqq C+\alpha \int_{0}^{t} \int_{-\infty}^{\infty}(1+\tau)^{\alpha-1} \eta_{*} d x d \tau+C \varepsilon(1+t)^{\alpha+1} \\
& \leqq C+\alpha \int_{0}^{t} \int_{-\infty}^{\infty}(1+\tau)^{\alpha-1}\left(\frac{\left(m^{\varepsilon}\right)^{2}}{2 \rho^{\varepsilon}}+C_{7} y_{x}^{2}\right) d x d \tau+C \varepsilon(1+t)^{\alpha+1} \\
& \leqq C+\alpha \int_{0}^{t} \int_{-\infty}^{\infty}(1+\tau)^{\alpha-1} \frac{\left(m^{\varepsilon}\right)^{2}}{2 \rho^{\varepsilon}} d x d \tau+C \varepsilon(1+t)^{\alpha+1}
\end{aligned}
$$

and

$$
\int_{0}^{t} \int_{-\infty}^{\infty}(1+t)^{\alpha-1} \frac{\left(\bar{m}^{\varepsilon}\right)^{2}}{2 \bar{\rho}^{\varepsilon}} d x d \tau \leqq C \int_{0}^{t}(1+\tau)^{\alpha-1-\frac{1}{2}} d \tau \leqq C .
$$

By (3.13) and Lemma 3.2, we get

$$
\begin{aligned}
& \int_{-\infty}^{\infty}(1+t)^{\alpha} \eta_{*} d x+\int_{0}^{t} \int_{-\infty}^{\infty}(1+\tau)^{\alpha}\left(\frac{\left(m^{\varepsilon}\right)^{2}}{\rho^{\varepsilon}}-\frac{\left(\bar{m}^{\varepsilon}\right)^{2}}{\bar{\rho}^{\varepsilon}}\right) d x d \tau \\
& \leqq C+C \varepsilon(1+t)^{\alpha+1}
\end{aligned}
$$


To obtain the decay rates, we need to analyze carefully the term

$$
\int_{0}^{t} \int_{-\infty}^{\infty}(1+t)^{\alpha}\left(\frac{\left(m^{\varepsilon}\right)^{2}}{\rho^{\varepsilon}}-\frac{\left(\bar{m}^{\varepsilon}\right)^{2}}{\bar{\rho}^{\varepsilon}}\right) d x d \tau .
$$

We note that

$$
\frac{\left(m^{\varepsilon}\right)^{2}}{\rho^{\varepsilon}}-\frac{\left(\bar{m}^{\varepsilon}\right)^{2}}{\bar{\rho}^{\varepsilon}}=Q+\frac{2 \bar{m}^{\varepsilon} z}{\bar{\rho}^{\varepsilon}}+\frac{\left(\bar{m}^{\varepsilon}\right)^{2}}{\left(\bar{\rho}^{\varepsilon}\right)^{2}} y_{x} .
$$

It is easy to see that

$$
\begin{aligned}
\int_{0}^{t} \int_{-\infty}^{\infty}(1+\tau)^{\alpha}\left(\bar{m}^{\varepsilon}\right)^{2} y_{x} d x d \tau \leqq & C \int_{0}^{t} \int_{-\infty}^{\infty}(1+\tau)^{2 \alpha}\left(\bar{m}^{\varepsilon}\right)^{4} d x d \tau \\
& +C \int_{0}^{t} \int_{-\infty}^{\infty} y_{x}^{2} d x d \tau \leqq C+C \varepsilon t
\end{aligned}
$$

We compute

$$
\begin{aligned}
(1+t)^{\alpha} \frac{\bar{m}^{\varepsilon}}{\bar{\rho}^{\varepsilon}} z= & (1+t)^{\alpha} \frac{\bar{m}^{\varepsilon}}{\bar{\rho}^{\varepsilon}}\left(y_{t}-\varepsilon y_{x x}\right) \\
= & \varepsilon(1+t)^{\alpha}\left(\frac{\bar{m}^{\varepsilon}}{\bar{\rho}^{\varepsilon}}\right)_{x} y_{x}+\left((1+t)^{\alpha} \frac{\bar{m}^{\varepsilon}}{\bar{\rho}^{\varepsilon}} y\right)_{t} \\
& -(1+t)^{\alpha}\left(\frac{\bar{m}^{\varepsilon}}{\bar{\rho}^{\varepsilon}}\right)_{t} y-\alpha(1+t)^{\alpha-1} \frac{\bar{m}^{\varepsilon}}{\bar{\rho}^{\varepsilon}} y+(\cdots)_{x} .
\end{aligned}
$$

The terms on the right-hand side of (3.31) can be controlled as follows:

$$
\begin{aligned}
& \varepsilon \int_{0}^{t} \int_{-\infty}^{\infty}(1+\tau)^{\alpha}\left(\frac{\bar{m}^{\varepsilon}}{\bar{\rho}^{\varepsilon}}\right)_{x} y_{x} d x d \tau \\
& \quad \leqq C \varepsilon \int_{0}^{t} \int_{-\infty}^{\infty}(1+\tau)^{2 \alpha}\left(\bar{\rho}_{x}^{\varepsilon}\right)^{2} d x d \tau+\varepsilon \int_{0}^{t} \int_{-\infty}^{\infty} y_{x}^{2} d x d \tau \\
& \quad \leqq C+C \varepsilon t .
\end{aligned}
$$

For the third term, we have

$$
\begin{aligned}
& \int_{0}^{t} \int_{-\infty}^{\infty}(1+\tau)^{\alpha}\left(\frac{\bar{m}^{\varepsilon}}{\bar{\rho}^{\varepsilon}}\right)_{t} y d x d \tau \\
& \quad \leqq \int_{0}^{t} \int_{-\infty}^{\infty}(1+t)^{2 \alpha+1+\delta}\left(\bar{\rho}_{x t}^{\varepsilon}\right)^{2} d x d \tau+\int_{0}^{t} \int_{-\infty}^{\infty}(1+\tau)^{-1-\delta} y^{2} d x d \tau \\
& \quad \leqq C+\int_{0}^{t}(1+\tau)^{2 \alpha+1+\delta-2-\frac{1}{2}} d \tau+C \varepsilon t \\
& \quad \leqq C+C \varepsilon t, \text { if } 0<\delta<\frac{1}{2}-2 \alpha
\end{aligned}
$$

For the fourth term, we get

$$
\begin{aligned}
\int_{0}^{t} \int_{-\infty}^{\infty}(1+\tau)^{\alpha-1} \frac{\bar{m}^{\varepsilon}}{\bar{\rho}^{\varepsilon}} y d x d \tau & \leqq \int_{0}^{t}(1+\tau)^{2 \alpha-2+1+\delta-\frac{1}{2}} d \tau+C+C \varepsilon t \\
& \leqq C+C \varepsilon t, \text { if } 0<\delta<\frac{1}{2}-2 \alpha
\end{aligned}
$$


For the second term, we use (3.24) to derive the following estimate

$$
\begin{aligned}
& \int_{0}^{t} \int_{-\infty}^{\infty}\left((1+\tau)^{\alpha} \frac{\bar{m}^{\varepsilon}}{\bar{\rho}^{\varepsilon}} y\right)_{t} d x d \tau \\
& \quad \leqq C+C \varepsilon t+\int_{-\infty}^{\infty}(1+t)^{2 \alpha}\left(\bar{\rho}_{x}^{\varepsilon}\right)^{2} d x \leqq C+C \varepsilon t .
\end{aligned}
$$

Therefore, from (3.28)-(3.35), we have

$$
(1+t)^{\alpha} \int_{-\infty}^{\infty} \eta_{*} d x+\int_{0}^{t} \int_{-\infty}^{\infty}(1+t)^{\alpha} Q d x d \tau \leqq C+C \varepsilon(1+t)^{\alpha+1}
$$

which implies

$$
(1+t)^{\alpha} \int_{-\infty}^{\infty} \eta_{*} d x \leqq C+C \varepsilon(1+t)^{\alpha+1}
$$

because $Q \geqq 0$. Notice that

$$
\eta_{*} \geqq \frac{m^{\varepsilon^{2}}}{2 \rho^{\varepsilon}}+\sigma y_{x}^{2}
$$

and we have

$$
(1+t)^{\alpha} \int_{-\infty}^{\infty} \frac{m^{\varepsilon^{2}}}{\rho^{\varepsilon}} d x \leqq C+C \varepsilon(1+t)^{\alpha+1} .
$$

On the other hand, by (3.6), we calculate

$$
\begin{aligned}
&(1+t)^{\alpha} \int_{-\infty}^{\infty} \frac{z^{2}}{\bar{\rho}^{\varepsilon}} d x \\
& \leqq C(1+t)^{\alpha} \int_{-\infty}^{\infty} \frac{\left(m^{\varepsilon}\right)^{2}}{\rho^{\varepsilon}} d x+(1+t)^{\alpha} \int_{-\infty}^{\infty} \frac{\left(\bar{m}^{\varepsilon}\right)^{2}}{\bar{\rho}^{\varepsilon}} d x \\
&+\int_{-\infty}^{\infty} \frac{2 \bar{m}^{\varepsilon}}{\bar{\rho}^{\varepsilon}} z(1+t)^{\alpha} d x \\
& \leqq C+C \varepsilon(1+t)^{\alpha+1}
\end{aligned}
$$

Due to the fact that $0<C_{1} \leqq \bar{\rho}^{\varepsilon} \leqq C_{2}$, we have

$$
(1+t)^{\alpha} \int_{-\infty}^{\infty} z^{2} d x \leqq C+C \varepsilon(1+t)^{\alpha+1} .
$$

This, together with (3.36), yields

$$
(1+t)^{\alpha} \int_{-\infty}^{\infty}\left(z^{2}+y_{x}^{2}\right) d x \leqq C+C \varepsilon(1+t)^{\alpha+1} .
$$


Lemma 3.5. Under the conditions of Theorem 1, there is a positive constant $C$ and an $\alpha<\frac{1}{4}$ independent of time such that, for any $t \geqq 0$,

$$
(1+t)^{\alpha} \int_{-\infty}^{\infty}\left[(\rho-\bar{\rho})^{2}+(m-\bar{m})^{2}\right] d x \leqq C .
$$

Since $|\rho-\bar{\rho}|$ and $|m-\bar{m}|$ are uniformly bounded, it follows that

$$
(1+t)^{\alpha} \int_{-\infty}^{\infty}|\rho-\bar{\rho}|^{p}+|m-\bar{m}|^{p} d x \leqq C .
$$

When $\rho_{-}=\rho_{+}>0$, we can get a better convergence rate. In fact, by the uniqueness theorem of strictly parabolic equation, the solution $\bar{\rho}^{\varepsilon}=\rho_{-}$, and $\bar{m}^{\varepsilon}=-P\left(\bar{\rho}^{\varepsilon}\right)_{x}=0$. Thus, (3.11) becomes

$$
\frac{d}{d t} \int_{-\infty}^{\infty} \eta_{*}(x, t) d x+\int_{-\infty}^{\infty} \frac{m^{\varepsilon 2}}{\rho^{\varepsilon}} d x \leqq 0,
$$

due to $\int_{-\infty}^{\infty} P^{\prime}\left(\rho_{-}\right)\left(\rho^{\varepsilon}-\rho_{-}\right) d x=0$.

Multiplying (3.45) by $1+t$, we have

$$
(1+t) \frac{d}{d t} \int_{-\infty}^{\infty} \eta_{*}(x, t) d x+(1+t) \int_{-\infty}^{\infty} \frac{m^{\varepsilon^{2}}}{\rho^{\varepsilon}} d x \leqq 0 .
$$

By the argument above, (3.27) is changed into

$$
\int_{-\infty}^{\infty}(1+t) \eta_{*} d x+\int_{0}^{t} \int_{-\infty}^{\infty}(1+\tau) \frac{\left(m^{\varepsilon}\right)^{2}}{\rho^{\varepsilon}} d x d \tau \leqq C,
$$

which implies

$$
(1+t) \int_{-\infty}^{\infty} \eta_{*} d x \leqq C
$$

This, together with Lemma 3.1 and (3.40), implies

$$
(1+t) \int_{-\infty}^{\infty}\left(z^{2}+y_{x}^{2}\right) d x \leqq C .
$$

Hence we have

Lemma 3.6. Under the conditions of Theorem 1, if $\rho_{-}=\rho_{+}>0$, then there is a positive constant $C$ independent of time such that, for any $t \geqq 0$,

$$
(1+t) \int_{-\infty}^{\infty}\left(z^{2}+y_{x}^{2}\right) d x \leqq C .
$$

Therefore Theorem 1 is proved by Lemmas 3.5 and 3.6. 


\section{References}

1. ARonson, D.G.: The porous media equations. In Nonlinear Diffusion Problem. Lecture Notes in Math., Vol. 1224, (A. FASANO, M. PRIMICERIO, Eds) Springer-Verlag, Berlin, 1986

2. Brezis, H., Crandall, M.: Uniqueness of solutions of the initial-value problem for $u_{t}-\Delta \phi(u)=0$. J. Math. pures et. appl. 58, 153-163 (1979)

3. DAFERMOS, C.M.: A system of hyperbolic conservation laws with frictional damping. Z. Angew. Math. Phys. 46, 294-307 (1995)

4. Ding, X., Chen, G., LuO, P.: Convergence of the Lax-Friedrichs scheme for isentropic gas dynamics (I)(II). Acta Math. Sci 5, 483-500, 501-540 (1985)

5. Ding, X., Chen, G., LuO, P.: Convergence of the fractional step Lax-Friedrichs and Godunov scheme for isentropic system of gas dynamics. Commun. Math. Phys 121, 63-84 (1989)

6. DiPernA, R.J.: Convergence of approximate solutions of conservation laws. Arch. Rational Mech. Anal. 82, 27-70 (1983)

7. DuYn, C.J., Peletier, L.A.: A class of similary solutions of the nonlinear diffusion equations. Nonlinear Analysis, TMA 1, 223-233 (1977)

8. HSIAO, L.: Quasilinear hyperbolic systems and dissipative mechanisms. World Scientific 1997

9. HSIAO, L., LIU, T.P.: Convergence to nonlinear diffusion waves for solutions of a system of hyperbolic conservation laws with damping. Comm. Math. Phys. 143, 599-605 (1992)

10. HsiaO, L., LIU, T.P.: Nonlinear diffusive phenomena of nonlinear hyperbolic systems. Chin. Ann. of Math. Ser. B 14, 465-480 (1993)

11. HSIAO, L., LUO, T.: Nonlinear diffusive phenomena of entropy weak solutions for a system of quasilinear hyperbolic conservation laws with damping. Q. Appl. Math. 56, 173-198 (1998)

12. HsiaO, L., PAN, R.H.: The damped p-system with boundary effects. Contemporary Mathematics 255, 109-123 (2000)

13. HSIAO, L., TANG, S.Q.: Construction and qualitative behavior of solutions for a system of nonlinear hyperbolic conservation laws with damping. Q. Appl. Math. 53, 487-505 (1995)

14. HSIAO, L., TANG, S.Q.: Construction and qualitative behavior of solutions of perturbated Riemann problem for the system of one-dimensional isentropic flow with damping. J. Differential Equations 123, 480-503 (1995)

15. HuANG, F.M., PAN, R.H.: Asymptotic behavior of the solutions to the damped compressible Euler equations with vacuum. Preprint (2000)

16. KAMIN, S.: Source-type solutions for equations of nonstationary filtration. J. Math. Anal. Appl. 64, 263-276 (1978)

17. LiU, T.P.: Compressible flow with damping and vacuum Japan. J. Appl. Math 13, 25-32 (1996)

18. Lions, P.L., Perthame, B., Souganidis, P.E.: Existence of entropy of solutions for the hyperbolic systems of isentropic gas dynamics in Eulerian and Lagrangian coordinates. Comm. Pure Appl. Math. 49, 599-638 (1996)

19. Luskin, M., TEMPLE, B.: The existence of a global weak solution to the nonlinear water-hammer problem. Comm. Pure Appl. Math. 35, 697-735 (1982)

20. LIU, T.P., YANG, T.: Compressible Euler equations with vacuum. J. Differential Equations 140, 223-237 (1997)

21. LiU, T.P., YANG, T.: Compressible flow with vacuum and physical singularity. Methods Appl. Anal. 7, 495-509 (2000)

22. LUO, T., YANG, T.: Interaction of elementary waves for compressible Euler equations with frictional damping. J. Differential Equations 161, 42-86 (2000)

23. NISHIDA, T.: Nonlinear hyperbolic equations and related topics in fluid dynamics. Publ. Math. D'Orsay 46-53 (1978) 
24. NISHIHARA, K.: Convergence rates to nonlinear diffusion waves for solutions of system of hyperbolic conservation laws with damping. J. Differential Equations 131, 171-188 (1996)

25. NISHIHARA, K., WANG, W., YANG, T.: $L_{p}$-convergence rate to nonlinear diffusion waves for $p$-system with damping. J. Differential Equations 161, 191-218 (2000)

26. NISHIHARA, K., YANG, T.: Boundary effect on asymptotic behavior of solutions to the $p$-system with damping. J. Differential Equations 156, 439-458 (1999)

27. PIERRE, M.: Uniqueness of solutions of $u_{t}-\Delta \phi(u)=0$ with inital datum a measure. Nonlinear Analysis, TMA 6, 175-187 (1982)

28. SERRE, D., XIAO, L.: Asymptotic behavior of large weak entropy solutions of the damped p-system. J. P. Diff. Eqns. 10, 355-368 (1997)

29. ZHAO, H.J.: Convergence to strong nonlinear diffusion waves for solutions of $p$-system with damping. J. Differential Equations 174, 200-236 (2001)

30. ZHENG, Y.S.: Global smooth solutions to the adiabatic gas dynamics system with dissipation terms Chinese. Ann. of Math. 17A, 155-162 (1996)

31. ZHU, C.J.: Convergence Rates to Nonlinear Diffusion Waves for Weak Entropy Solutions to $p$-System with Damping. Preprint (2000)

Institute of Applied Mathematics Academia Sinica

Beijing, China

and

Department of Mathematics

University of Michigan

Ann Arbor, MI 48109-1109

e-mail: panrh@umich.edu

(Accepted August 31, 2002)

Published online January 9, 2003 - (C) Springer-Verlag (2003) 